\title{
Electrically Injected InGaAsP Microdisk Lasers Heterogeneously Integrated on a Si-wafer
}

\author{
J. Van Campenhout ${ }^{1}$, Student Member IEEE, P. Rojo-Romeo ${ }^{2}$, D. Van Thourhout ${ }^{1}$, Member IEEE, \\ C. Seassal ${ }^{2}$, P. Regreny ${ }^{2}$, L. Di Cioccio ${ }^{3}$, J.M. Fedeli ${ }^{3}$, and R. Baets ${ }^{1}$, Senior Member IEEE. \\ ${ }^{1}$ Ghent University-IMEC, Department of Information Technology- \\ Sint-Pietersnieuwstraat 41, 9000 Gent, Belgium \\ ${ }^{2}$ Laboratoire d'Electronique, Microelectronique et Micro-systèmes, Ecole Centrale de Lyon, \\ 36 Avenue Guy de Collongue, 69134 Ecully cedex-France \\ ${ }^{3}$ CEA-DRT/LETI, 17 Rue des Martyrs, 38054 Grenoble cedex 9-France. \\ e-mail: joris.vancampenhout@intec.ugent.be
}

\begin{abstract}
We report on electrically pumped lasing in microdisk cavities etched in a thin InP-based membrane directly bonded on top of a silicon wafer. The microdisks were planarized by means of a benzocyclobutene (BCB) layer, into which contact windows were etched to perform current injection. The top metal contact is placed only in the centre of the disk, whereas the bottom contacting is done by means of a thin lateral InP contacting layer. In order to avoid large optical absorption in ptype contact layers, a tunnel junction was used in combination with two n-type contacts. Lasing was observed in pulsed regime at room temperature with current thresholds in the range 0.55 $1.1 \mathrm{~mA}$, for microdisks with a diameter in the range $6-9 \mu \mathrm{m}$.
\end{abstract}

Index Terms - InGaAsP, semiconductor laser, microdisk, benzocyclobutene, optical interconnect

\section{INTRODUCTION}

$\mathrm{F}_{\mathrm{b}}$ OR future generation electronic circuits, a severe bottleneck is expected on the global interconnect level. With decreasing device dimensions, it is increasingly difficult to keep propagation delays and power consumption acceptable. Therefore there is a need for radically different interconnect approaches and one of the most promising solutions is the use of an optical interconnect layer. A possible approach for a compact optical link is the use of a Silicon-onInsulator (SOI) passive waveguide layer [1] in combination with III-V semiconductor microlasers and microdetectors, which are defined in a III-V membrane bonded on top of the SOI-stack. A good candidate for the microlaser is the membrane microdisk laser. In this paper, we report on the lasing characteristics of electrically injected, thin membrane microdisk lasers integrated on a Si-wafer.

\section{Design AND FABRICATION ASPECTS}

An important design aspect of electrically injected, thin membrane laser devices is the epilayer heterostructure
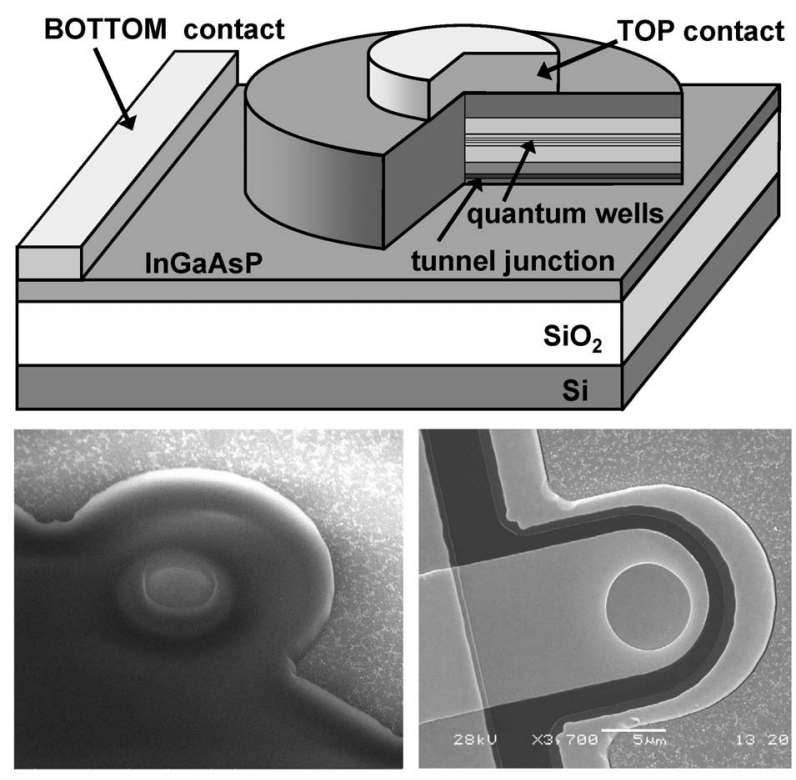

Fig. 1. Top: General layout of the membrane microdisk laser. Below: SEM-pictures after BCB opening (left) and after metallization (right).

composition: this should allow efficient carrier injection while preserving optical resonance quality. An important issue is how to contact the p-type layer of the pn-junction. In classic substrate lasers, this is done by means of a highly doped, lowbandgap semiconductor layer. However, these layers are very absorptive and thus not useable in a thin membrane. Therefore, we implemented a tunnel junction, in combination with a second n-type contact (see fig. 1). This tunnel junction consists of a reverse-biased Q1.2 $\mathrm{p}^{++} / \mathrm{n}++$-junction with layer thicknesses of only $20 \mathrm{~nm}$ and doping levels above $10^{19} \mathrm{~cm}^{-3}$. This type of tunnel junction can have low absorption losses in combination with a low electrical resistivity [2]. A second important aspect is the position of the metal contacts. They should be kept away form the optical field to avoid any excess absorption. In our design, the top contact is placed only in the centre of the microdisk, whereas the bottom contact is placed on a very thin n-type InP layer that extends laterally at the 
bottom of the microdisk. This bottom contact layer can cause optical leakage if it is too thick. However, a 3D FDTD analysis revealed that these structures can support whispering gallery modes with quality factors over 10000 for a $50 \mathrm{~nm}$ thick bottom contact layer, a disk diameter $D$ of $4 \mu \mathrm{m}$ and a total disk thickness of $500 \mathrm{~nm}$.

First, the laser heterostructure including three compressively strained InAsP quantum wells and the tunnel junction was grown by molecular beam epitaxy (MBE) on a 2 inch InP wafer. This wafer was bonded onto a $\mathrm{Si}$ wafer by molecular bonding (for more details, see [3]). After substrate removal, the microdisks were defined by optical lithography and were etched into the $420 \mathrm{~nm}$-thick bonded membrane by reactive ion etching (RIE) using a Ti hard mask. The RIE etch was incomplete, leaving a thin bottom contact membrane of about $80 \mathrm{~nm}$. These structures were covered with a benzocyclobutene (BCB) film, in which contact windows were etched before depositing the top and bottom metal contact. Au-based contacts were deposited and fast-alloyed at $400^{\circ} \mathrm{C}$.

\section{Measurement Results}

Electrically injected lasing was observed for microdisks with diameters in the range $6-9 \mu \mathrm{m}$. The P-I curve and lasing spectrum for a disk with diameter of $6 \mu \mathrm{m}$ is depicted in fig. 2 and reveals a threshold current as low as $0.55 \mathrm{~mA}$, and a lasing wavelength of $1570 \mathrm{~nm}$. For all diameters, the microdisks lase only in the radially fundamental whispering gallery modes, due to the presence of the top metal contact in the centre of the disk. For smaller top metal contacts, we also observed lasing for radially higher order modes. The current threshold as a function of diameter is also depicted in fig 2: for disk diameters smaller than $6 \mu \mathrm{m}$, we failed to open the top contact window in the BCB-layer. Lasing was only obtained in pulsed regime, due to the high electrical resistance of the device in combination with a poor heat sinking ability. Indeed, the voltage drop at threshold varies between $5-7 \mathrm{~V}$, mainly due to the non-optimal tunnel junction and non-optimal n-type metal contacts. A thermal analysis of the microdisk laser yields a thermal resistance of about $10^{4} \mathrm{~K} / \mathrm{W}$ for $D=6 \mu \mathrm{m}$, what results in serious self heating. Future work includes coupling to a passive SOI-waveguide and improvement of the tunnel junction, metal contacts and etching quality.
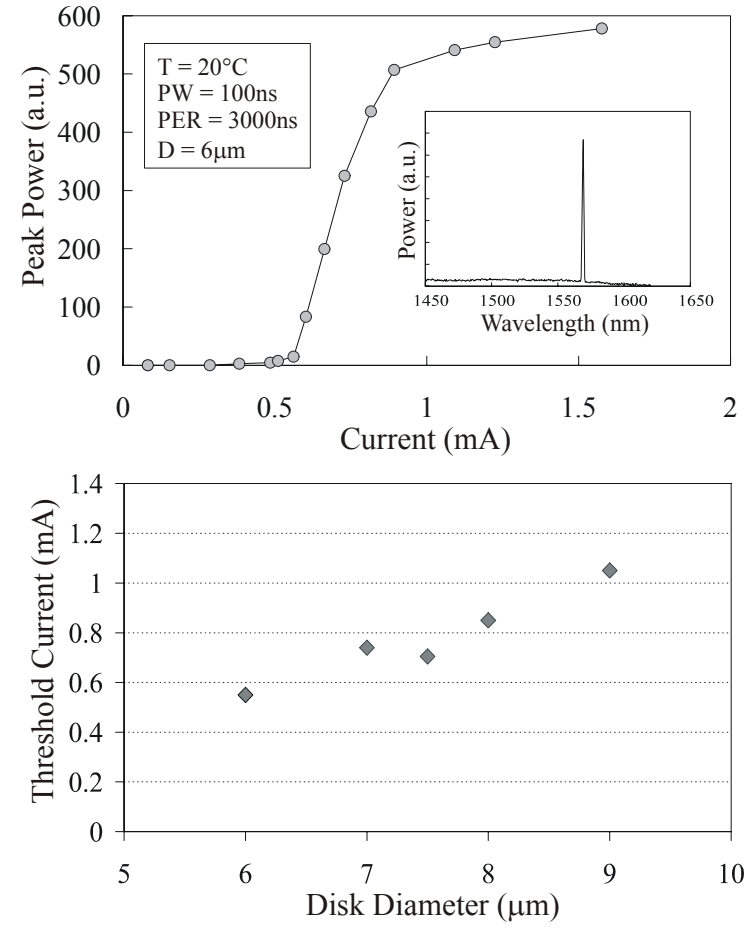

Fig. 2. Top: Light output vs. current for a disk diameter of $6 \mu \mathrm{m}$. Inset: lasing spectrum for $0.75 \mathrm{~mA}$. Below: threshold current vs. disk diameter.

\section{ACKNOWLEDGMENT}

The work of J. Van Campenhout was supported by the Research Foundation - Flanders (FWO-Vlaanderen). Part of this work was performed in the context of the EU-funded project PICMOS.

\section{REFERENCES}

[1] W. Bogaerts, R. Baets, P. Dumon, V. Wiaux, S. Beckx, D.Taillaert, B. Luyssaert, J. Van Campenhout, P. Bienstman and D. Van Thourhout, "Nanophotonic waveguides in silicon-on-insulator fabricated with CMOS technology", Journ. of Lightwave Technology, Vol. 23(1), pp. 401 - 412, Jan 2005

[2] J. Boucart, C. Starck, F. Gaborit, A. Plais, N. Bouché, E. Derouin, J. C. Remy, J. Bonnet-Gamard, L. Goldstein, C. Fortin, D. Carpentier, P. Salet, F. Brillouet, and J. Jacquet, "Metamorphic DBR and TunnelJunction Injection:A CW RT Monolithic Long-Wavelength VCSEL", Journ. Sel. Top. Quant. Elec., Vol. 5(3), pp. 520 - 529, May 1999

[3] C. Seassal, P. Rojo-Romeo, X. Letartre, P. Viktorovitch, G. Hollinger, E. Jalaguier, S. Pocas and B. Aspar, "InP microdisk lasers on silicon wafer:

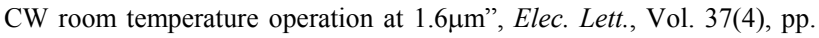
$222-223$, Feb 2001 
